\title{
Approximate analysis of thin beam with variable prestress on elastic foundation
}

\author{
Ogunyebi S.N \\ Department of Mathematical Sciences, Ekiti State University, Ado-Ekiti, Ekiti State, Nigeria.
}

\begin{abstract}
In this article, the effect of variable prestress on the behavior of thin beam on constant elastic foundation is presented. The moving load is distributed over the entire span of the beam and governs by fourth order partial differential equation. It is shown from the numerical analysis that the higher values of axial force $N$, the lower the amplitude response of the beam with variable prestress. The same argument goes for foundation rigidity $K_{b}$. Results in plotted curves indicate that resonance is reached earlier in moving mass solution than moving force solution.
\end{abstract}

Keywords: Distributed load, Prestress, Resonance, Response, Thin beam.

\section{Introduction}

Study pertaining to the subject of moving load on elastic foundation has attracted several researchers in the field of dynamics of structures. Extensive work has been done in this area especially when the structural members have uniform cross-section.

Many authors have widely studied behavior of structures (beam or plate) under moving loads [1,2,3,4]. In most of these studies, the assumption has been that prestress in beams have been of uniform type all through the length of the structural members. Cases where the effects of axial force is of non-uniform on the frequencies has been neglected especially when the loads on the structure are in the distributed form. In most of the existing literature in dynamics of structure, moving loads have been idealized as moving concentrated loads which acts at a certain point on the structure and along a single line in space $[5,6]$. The problem of traverse oscillations of beams under the action of moving loads for the general case of any arbitrary prescribed law of motion was solved by Lowan [7] using Green's functions. Furthermore, Timoshenko [8] and Timoshenko et al [9] considered a case when a pulsating force is moving along a beam with a constant velocity using the principle of virtual work.

Omolofe and Ajibola [10] considered, the transverse motions of thin beam with variable prestress under moving loads using Generalized Galekin's method and the modified Asymptotic method of Struble to obtain analytical solutions. They considered in their study moving concentrated load system on constant foundation.

It is observed that beams having variable prestress and resting on elastic foundation under moving distributed loads have received scanty attention. Therefore, since prestress varies from a point to another along a structural member (beam or plate), this present work investigated the effects of variable axial force on the dynamic response to moving distributed load resting on a constant elastic foundation.

\section{Statement Of The Problem}

One of the well-known models in the family of beam's model is the thin beam theory (B-E beam theory). According to this theory, the length of each section is much greater than the height of each section and the shear and rotatory inertia effects are ignored.

Consider a thin beam of elastic material with modulus of elasticity $E$, moment of inertia $J$, the transverse displacement $V(x, t)$, constant mass per unit length, the foundation stiffness $K_{b}$, the position coordinate in the axial direction $x$, time $t$, the flexural rigidity $E J$ and the variable axial force $N(x)$.

$$
E J \frac{\partial^{4}}{\partial x^{4}} V(x, t)+\mu \frac{\partial^{2}}{\partial t^{2}} V(x, t)-\frac{\partial}{\partial x}\left[N(x) \frac{\partial}{\partial x} V(x, t)\right]+K_{b} V(x, t)=Q_{k}(x, t)\left[1-\frac{\nabla^{*}}{g} V(x, t)\right]
$$

where $\frac{\partial}{\partial x}$ is the partial derivative with respect to $x$. Since the thin beam has simple support at both ends, the pertinent boundary conditions 
$V(0, t)=0=V(L, t), \quad \frac{\partial^{2}}{\partial x^{2}} V(0, t)=0=\frac{\partial^{2}}{\partial x^{2}} V(L, t)$

The initial conditions(IC's) is given as

$V(x, 0)=0=\frac{\partial}{\partial t} V(x, 0)$

The moving force $Q_{k}(x, t)$ and operator $\nabla^{*}$ is as well defined in $[6,10]$

Let the variable axial force be of the form

$N(x)=N_{0}\left(1+\sin \frac{\pi x}{L}\right)^{3}$

where $N_{0}$ is the variable value of the axial force.

Substituting equations (4) into equation (1), after some rearrangement, the governing equation can be written as $E J \frac{\partial^{4}}{\partial x^{4}} V(x, t)+\mu \frac{\partial^{2}}{\partial t^{2}} V(x, t)-\frac{\partial}{\partial x}\left[N(x) \frac{\partial}{\partial x} V(x, t)\right]+K_{b} V(x, t)$

$+M H(x-c t)\left[\frac{\partial^{2}}{\partial t^{2}} V(x, t)+2 c \frac{\partial^{2}}{\partial x \partial t} V(x, t)+c^{2} \frac{\partial^{2}}{\partial x^{2}} V(x, t)\right]=\sum_{m=1}^{N} M g H(x-c t)$

Further simplification of equation (5) yields,

$$
\begin{aligned}
& E J \frac{\partial^{4}}{\partial x^{4}} V(x, t)+\mu \frac{\partial^{2}}{\partial t^{2}} V(x, t)-\frac{\partial}{\partial x}\left[N_{0}\left(\frac{5}{2}+\frac{15}{4} \sin \frac{\pi x}{L}-\frac{3}{2} \cos \frac{2 \pi x}{L}-\sin \frac{3 \pi x}{L}\right) \frac{\partial}{\partial x} V(x, t)\right] \\
& +K_{b} V(x, t)+M H(x-c t)\left[\frac{\partial^{2}}{\partial t^{2}} V(x, t)+2 c \frac{\partial^{2}}{\partial x \partial t} V(x, t)+c^{2} \frac{\partial^{2}}{\partial x^{2}} V(x, t)\right]=\sum_{m=1}^{N} M g H(x-c t)
\end{aligned}
$$

which is non-homogenous partial differential equation of the dynamical system with variable and singular coefficient.

\section{Analytical Approximate Solution}

It is evidently seen that a closed form solution of equation (6) does not exist. To this end, the Galerkin's method described in Oni and Ogunyebi [6] is employed to reduce the partial differential to a sequence of ordinary differential equation. This is defined

$$
V(x, t)=\sum_{m=1}^{\infty} W_{m}(t) U_{n}(x)
$$

where $U_{n}(x)$ is chosen such that the desired boundary conditions are satisfied.

Substituting equation (7) into (6) one obtains

$$
\begin{aligned}
& \sum_{m=1}^{n}\left\{E J \frac{m^{4} \pi^{4}}{L^{4}} \sin \frac{m \pi}{L}+\mu \sin \frac{m \pi}{L} \ddot{W}_{m}(t)+N_{0} \frac{m \pi}{4 L}\left(10 \frac{m \pi}{L} \sin \frac{m \pi x}{L}-15 \frac{\pi}{L} \cos \frac{\pi x}{L} \cos \frac{m \pi x}{L}\right.\right. \\
& +15 \frac{m \pi}{L} \sin \frac{\pi x}{L} \cos \frac{m \pi x}{L}-12 \frac{\pi}{L} \sin \frac{2 \pi x}{L} \cos \frac{m \pi x}{L}-6 \cos \frac{2 \pi x}{L} \sin \frac{m \pi x}{L}+3 \frac{\pi}{L} \cos \frac{3 \pi x}{L} \cos \frac{m \pi x}{L} \\
& \left.-\frac{m \pi x}{L} \sin \frac{3 \pi x}{L} \sin \frac{m \pi x}{L}+K_{b} \sin \frac{m \pi x}{L}\right) W_{m}(t)+M\left[H(x-c t) \ddot{W}_{m}(t) \sin \frac{m \pi x}{L}\right. \\
& \left.\left.+2 c \frac{m \pi}{L} H(x-c t) \dot{W}_{m}(t) \cos \frac{m \pi x}{L}-c^{2} \frac{m^{2} \pi^{2}}{L^{2}} H(x-c t) W_{m}(t) \sin \frac{m \pi x}{L}\right]\right\}-\sum_{m=1}^{n} M g H(x-c t)=0
\end{aligned}
$$

In order to determine $W_{m}(t)$ it is required that the expression on the left hand side of equation (8) be orthogonal to the function $U_{k}(x)$

Therefore, equation (8) becomes, 


$$
\begin{aligned}
& \sum_{m=1}^{n}\left\{B_{m 1} \ddot{W}_{m}(t)+\left(B_{m 2}+B_{m 3}\right) W_{m}(t)+\frac{M}{\mu}\left[B_{m a}(m, t) \ddot{W}_{m}(t)+2 c \frac{m \pi}{L} B_{m b}(m, t) \dot{W}_{m}(t)\right.\right. \\
& \left.\left.-C^{2} \frac{m^{2} \pi^{2}}{L^{2}} B_{m c}(m, t) W_{m}(t)\right]\right\}=\frac{M g}{\mu} B_{m d}(m, t)
\end{aligned}
$$

where

$$
\begin{gathered}
B_{m 1}=\int_{0}^{L} \sin \frac{m \pi x}{L} \sin \frac{k \pi x}{L} d x \quad ; \quad B_{m 2}=\frac{E J}{\mu} \frac{m^{4} \pi^{4}}{L^{4}} \int_{0}^{L} \sin \frac{m \pi x}{L} \sin \frac{k \pi x}{L} d x \\
B_{m 3}=F_{a}-F_{b}+F_{c}-F_{d}-F_{e}+F_{f}-F_{g}+F_{h} ; B_{m a}(m, t)=\int_{0}^{L} H(x-c t) \sin \frac{m \pi x}{L} \sin \frac{k \pi x}{L} d x \\
B_{m b}(m, t)=\int_{0}^{L} H(x-c t) \cos \frac{m \pi x}{L} \sin \frac{k \pi x}{L} d x ; B_{m c}(m, t)=\int_{0}^{L} H(x-c t) \sin \frac{m \pi x}{L} \sin \frac{k \pi x}{L} d x \\
B_{m d}(m, t)=\int_{0}^{L} H(x-c t) \sin \frac{k \pi x}{L} d x
\end{gathered}
$$

and

$$
\begin{gathered}
F_{a}=\frac{5 N_{0} m^{2} \pi^{2}}{2 L^{2} \mu} \int_{0}^{L} \sin \frac{m \pi x}{L} \sin \frac{k \pi x}{L} d x ; F_{b}=\frac{15 N_{0} m \pi^{2}}{4 L^{2}} \int_{0}^{L} \cos \frac{\pi x}{L} \cos \frac{m \pi x}{L} \sin \frac{k \pi x}{L} d x \\
F_{c}=\frac{15 N_{0} m \pi^{2}}{4 L^{2}} \int_{0}^{L} \sin \frac{\pi x}{L} \sin \frac{\pi x}{L} \sin \frac{k \pi x}{L} d x ; F_{d}=\frac{3 N_{0} m \pi^{2}}{4} \int_{0}^{L} \sin \frac{2 \pi x}{L} \cos \frac{m \pi x}{L} \sin \frac{k \pi x}{L} d x \\
F_{e}=\frac{3 m \pi N_{0}}{2 L} \int_{0}^{L} \cos \frac{2 \pi x}{L} \sin \frac{m \pi x}{L} \sin \frac{k \pi x}{L} d x ; F_{f}=\frac{3 m \pi^{2} N_{0}}{4 L^{2}} \int_{0}^{L} \cos \frac{3 \pi x}{L} \cos \frac{m \pi x}{L} \sin \frac{k \pi x}{L} d x \\
F_{g}=\frac{N_{0} m^{2} \pi^{2}}{4 L} \int_{0}^{L} \sin \frac{3 \pi x}{L} \sin \frac{m \pi x}{L} \sin \frac{k \pi x}{L} d x ; F_{h}=K_{b} \int_{0}^{L} \sin \frac{m \pi x}{L} \sin \frac{k \pi x}{L} d x
\end{gathered}
$$

The Heaviside function is defined in terms of Fourier sine series

$$
H(x-c t)=\frac{1}{4}+\frac{1}{\pi} \sum_{n=0}^{\infty} \sin \left[\frac{(2 n+1) \pi(x-c t)}{2 n+1}\right]
$$

Substituting equation (12) into equation (9), after some simplification and rearrangement takes the form

$$
\begin{aligned}
& \sum_{m=1}^{n}\left\{B_{m 1} \ddot{W}_{m}(t)+\left(B_{m 2}+B_{m 3}\right) W_{m}(t)+\gamma^{*}\left[\left(H_{a} / 4+\frac{1}{\pi} \sum_{n=0}^{\infty} \cos \frac{(2 n+1)}{2 n+1} \pi c t H_{b}-\frac{1}{\pi} \sum_{n=0}^{\infty} \sin \frac{(2 n+1)}{2 n+1} \pi c t H_{c}\right) \ddot{W}_{m}(t)\right.\right. \\
& \quad+2 c\left(H_{d} / 4+\frac{1}{\pi} \sum_{n=0}^{\infty} \cos \frac{(2 n+1)}{2 n+1} \pi c t H_{e}-\frac{1}{\pi} \sum_{n=0}^{\infty} \sin \frac{(2 n+1)}{2 n+1} \pi c t H_{f}\right) \dot{W}_{m}(t) \\
& \left.+c^{2}\left(\frac{H_{g}}{4} / 4+\frac{1}{\pi} \sum_{n=0}^{\infty} \cos \frac{(2 n+1)}{2 n+1} \pi c t H_{h}-\frac{1}{\pi} \sum_{n=0}^{\infty} \sin \frac{(2 n+1)}{2 n+1} \pi c t H_{i}\right) W_{m}(t)\right] \\
& =\frac{M g}{\mu m \pi}\left[-\cos m \pi+\cos \frac{m \pi c t}{L}\right]
\end{aligned}
$$

where

$\gamma^{*}=\frac{M}{\mu L}$

Equation (13) is called the transformed equation of our dynamical system. Two special cases of equation (13) are considered to give analytical solution of moving force and moving mass respectively in the distributed sense of the structural element [6].

\subsection{Moving distributed force case}

Setting the inertia term effect of equation (13) to zero and considering only a mass $M$ moving with a constant velocity c, equation (13) is re-arrange to take the form 


$$
B_{m 1} \ddot{W}_{m}(t)+B_{m 4} W_{m}(t)=\frac{M g}{\mu m \pi}\left[-\cos m \pi+\cos \frac{m \pi c t}{L}\right]
$$

where

$$
B_{m 4}=B_{m 2}+B_{m 3}
$$

Further simplification yields

$$
\ddot{W}_{m}(t)+\alpha_{f} W_{m}(t)=\frac{M g}{\mu m \pi B_{m 1}}\left[-\cos m \pi+\cos \frac{m \pi c t}{L}\right]
$$

Where

$$
\alpha_{f}=\frac{B_{m 4}}{B_{m 1}}
$$

Using Laplace method [3,6] equation (17) can be arranged to give

$$
W_{m}(t)\left[S^{2}+\alpha_{f}^{2}\right]=B_{m 5}\left[\frac{S}{S^{2}+\theta_{m}^{2}}-\frac{f_{0}}{S}\right]
$$

where

$$
f_{0}=(-1)^{m}, \theta_{m}=\frac{m \pi c}{L} \text { and } B_{m 5}=\frac{M g}{\mu m \pi B_{m 1}}
$$

then

$$
W_{m}(t)=B_{m 5}\left[f_{a_{1}}-f_{a_{2}}\right]
$$

where

$$
\begin{aligned}
& f_{a_{1}}=\frac{S}{S^{2}+\theta_{m}^{2}} \cdot \frac{1}{S^{2}+\alpha_{f}^{2}} \\
& f_{a_{2}}=\frac{f_{0}}{S} \cdot \frac{1}{S^{2}+\alpha_{f}^{2}}
\end{aligned}
$$

Clearly,

$$
f_{a_{1}}=\frac{\cos \theta_{m} t-\cos \alpha_{f} t}{\alpha_{f}^{2}-\theta_{m}^{2}}
$$

and

$$
f_{a_{2}}=\frac{\left(1-\cos \alpha_{f} t\right)}{\alpha_{f}}
$$

Substituting values of $f_{a_{1}}$ and $f_{a_{1}}$ into equation (21), one obtain

$$
W_{m}(t)=B_{m 5}\left[\frac{\cos \theta_{m} t-\cos \alpha_{f} t}{\alpha_{f}^{2}-\theta_{m}^{2}}+\frac{\left(1-\cos \alpha_{f} t\right)}{\alpha_{f}}\right]
$$

which when inverted yields

$V(x, t)=\sum_{m=1}^{\infty} \frac{2 B_{m 5}}{m \pi}\left[\frac{\cos \theta_{m} t-\cos \alpha_{f} t}{\alpha_{f}^{2}-\theta_{m}^{2}}+\frac{\left(1-\cos \alpha_{f} t\right)}{\alpha_{f}}\right] \times \sin \frac{m \pi x}{L}$

Equation (27) is the traverse displacement response to moving distributed force moving at constant speed on constant elastic foundation.

\subsection{Moving distributed mass case}

In this section, the entire solution to equation (13) is sought when all terms of inertia effect of the moving load is considered i.e. $\gamma^{*} \neq 0$

To this end equation (13) is rearranged to take the form 


$$
\begin{aligned}
& \ddot{W}_{m}(t)+\alpha_{f}^{2} W_{m}(t)+\gamma^{*}\left[\left(H_{a} / 4+\frac{1}{\pi} \sum_{n=0}^{\infty} \cos \frac{(2 n+1)}{2 n+1} \pi c t H_{b}-\frac{1}{\pi} \sum_{n=0}^{\infty} \sin \frac{(2 n+1)}{2 n+1} \pi c t H_{c}\right) \ddot{W}_{m}(t)\right. \\
& +2 c\left(H_{d} / 4+\frac{1}{\pi} \sum_{n=0}^{\infty} \cos \frac{(2 n+1)}{2 n+1} \pi c t H_{e}-\frac{1}{\pi} \sum_{n=0}^{\infty} \sin \frac{(2 n+1)}{2 n+1} \pi c t H_{f}\right) \dot{W}_{m}(t) \\
& \left.+c^{2}\left(H_{g} / 4+\frac{1}{\pi} \sum_{n=0}^{\infty} \cos \frac{(2 n+1)}{2 n+1} \pi c t H_{h}-\frac{1}{\pi} \sum_{n=0}^{\infty} \sin \frac{(2 n+1)}{2 n+1} \pi c t H_{i}\right) W_{m}(t)\right] \\
& =\frac{M g}{\mu m \pi B_{m 1}}\left[-\cos m \pi+\cos \frac{m \pi c t}{L}\right]
\end{aligned}
$$

To solve equation (28), the method of Struble's technique discussed in Oni and Ogunyebi [6] is employed. This is so because an exact analytical solution to equation (28) is not possible.

Therefore equation (28) can now be written as

$$
\begin{aligned}
& \ddot{W}_{m}(t)+2 c \frac{\left(H_{d} / 4+\frac{1}{\pi} \sum_{n=0}^{\infty} \cos \frac{(2 n+1)}{2 n+1} \pi c t H_{e}-\frac{1}{\pi} \sum_{n=0}^{\infty} \sin \frac{(2 n+1)}{2 n+1} \pi c t H_{f}\right)}{\left[1+\gamma^{*}\left(H_{a} / 4+\frac{1}{\pi} \sum_{n=0}^{\infty} \cos \frac{(2 n+1)}{2 n+1} \pi c t H_{b}-\frac{1}{\pi} \sum_{n=0}^{\infty} \sin \frac{(2 n+1)}{2 n+1} \pi c t H_{c}\right)\right]_{m}(t)} \dot{W}^{2} \\
& +\frac{\alpha_{f}^{2}+\gamma^{*} c^{2}\left(H_{g} / 4+\frac{1}{\pi} \sum_{n=0}^{\infty} \cos \frac{(2 n+1)}{2 n+1} \pi c t H_{h}-\frac{1}{\pi} \sum_{n=0}^{\infty} \sin \frac{(2 n+1)}{2 n+1} \pi c t H_{i}\right)}{\left[1+\gamma^{*}\left(H_{a} / 4+\frac{1}{\pi} \sum_{n=0}^{\infty} \cos \frac{(2 n+1)}{2 n+1} \pi c t H_{b}-\frac{1}{\pi} \sum_{n=0}^{\infty} \sin \frac{(2 n+1)}{2 n+1} \pi c t H_{c}\right)\right]} \dot{W}_{m}(t) \\
& \left.=1+\gamma^{*}\left(H_{a} / 4+\frac{1}{\pi} \sum_{n=0}^{\infty} \cos \frac{(2 n+1)}{2 n+1} \pi c t H_{b}-\frac{1}{\pi} \sum_{n=0}^{\infty} \sin \frac{(2 n+1)}{2 n+1} \pi c t H_{c}\right)\right]
\end{aligned}
$$

The homogenous part of equation (29) is first treated and a modified frequency corresponding to the frequency of the free system due to the presence of moving distributed mass is sought. To this end, an equivalent free system operator defined by the modified frequency then replaces equation (29). To do this, we consider a parameter $\varepsilon_{m}<1$ for any arbitrary mass ratio $\gamma^{*}$ defined as

$$
\varepsilon_{m}=\frac{\gamma^{*}}{1+\gamma^{*}}
$$

so

$\gamma^{*}=\varepsilon_{m}\left[1+0\left(\varepsilon_{m}\right)+0\left(\varepsilon_{m}^{2}\right)+\cdots\right]$

since

$\frac{1}{\left[1+\varepsilon_{m}\left(H_{m m}(n)\right)\right]}=\left[1-\gamma^{*}\left(H_{m m}(n)\right)\right]+0\left(\varepsilon_{m}^{2}\right)$

where

$$
H_{m m}(n)=H_{a} / 4+\frac{1}{\pi} \sum_{n=0}^{\infty} \cos \frac{(2 n+1)}{2 n+1} \pi c t H_{b}-\frac{1}{\pi} \sum_{n=0}^{\infty} \sin \frac{(2 n+1)}{2 n+1} \pi c t H_{c}
$$

Therefore all the coefficients of $W_{m}(t)$ and its derivatives in equation (29) are written in terms of $\varepsilon_{m}$. And setting $\varepsilon_{m}$ to zero we obtain a situation corresponding to the case in which the inertia effect of the mass of the system is regarded as negligible is obtained. Then the solution is written in the form

$$
W_{m}(t)=f_{m m} \cos \left[\alpha_{f} t-\Omega_{f}(t)\right]
$$


where $f_{m m}$ and $\Omega_{f}$ are constants and $\alpha_{f}=\frac{B_{m 4}}{B_{m 1}}$

By Struble's technique, it is required that the solution of the homogenous part of equation (2.27) be written in the form

$$
W_{m}(t)=C_{m m}(t) \cos \left[\alpha_{f} t-\Omega_{f}(t)\right]+\varepsilon_{m} W_{1}(t)+0\left(\varepsilon_{m}^{2}\right)
$$

Substituting equations (35) and its derivatives into homogenous parts of equation (29) one obtains

$$
\begin{aligned}
& 2 \dot{C}_{m m}(t) \alpha_{f} \sin \left[\alpha_{f} t-\Omega_{f}(t)\right]+2 C_{m m}(t) \alpha_{f} \dot{\Omega}_{f}(t) \cos \left[\alpha_{f} t-\Omega_{f}(t)\right] \\
& -2 c \varepsilon_{m} H_{d} / 4 C_{m m}(t) \alpha_{f} \sin \left[\alpha_{f} t-\Omega_{f}(t)\right]-\varepsilon_{m} \alpha_{f}^{2} H_{a} / 4 C_{m m}(t) \cos \left[\alpha_{f} t-\Omega_{f}(t)\right] \\
& +\varepsilon_{m} H_{g} / 4 C_{m m}(t) \cos \left[\alpha_{f} t-\Omega_{f}(t)\right]=0
\end{aligned}
$$

It is noted that in equation (36), higher order terms $0\left(\varepsilon_{m}\right)$ and terms that do not contribute to the variational equations have been neglected. Thus setting the quotient of $\sin \left[\alpha_{f} t-\Omega_{f}(t)\right]$ and $\cos \left[\alpha_{f} t-\Omega_{f}(t)\right]$ to zero in equation (36) we obtain,

$$
2 \dot{C}_{m m}(t) \alpha_{f}-2 c \varepsilon_{m} H_{d} / 4 C_{m m}(t) \alpha_{f}=0
$$

and

$$
2 C_{m m}(t) \alpha_{f} \dot{\Omega}_{f}(t)-\varepsilon_{m} \alpha_{f}^{2} H_{a} / 4 C_{m m}(t)+\varepsilon_{m} H_{g} / 4 C_{m m}(t)=0
$$

from equation (37) we have

$$
C_{m m}(t)=f_{0} e^{C \varepsilon_{m} H_{d} / 4}
$$

and from (38)

$$
\Omega_{f}(t)=\frac{\varepsilon_{m}}{2 \alpha_{f}^{2} t}\left[\alpha_{f} H_{a} / 4-\alpha_{f}^{2} H_{g} / 4\right] t+\phi_{f}
$$

where $\phi_{f}$ is a constant

Substituting (39) and (40) into (35), we have

$$
W_{m}(t)=f_{0} e^{c \varepsilon_{m} H_{d} / 4} \cos \left[\alpha_{f f} t-\phi_{f}\right]
$$

Therefore when the mass effect of the particle is considered, the first approximation to the homogenous system is given as

where

$$
W_{m}(t)=C_{m m}(t) \cos \left[\alpha_{f f} t-\phi_{f}\right]
$$

$$
\alpha_{f f}=\alpha_{f}\left[1-\frac{\varepsilon_{m}}{2 \alpha_{f}^{2}}\left(\alpha_{f} H_{a} / 4-\alpha_{f}^{2} H_{g} / 4\right)\right]
$$

is the modified frequency corresponding to the frequency of the free system due to the presence of the moving distributed mass. In view (41), the homogenous part of the equation (29) can be written as

$$
\frac{d^{2}}{d t^{2}} W_{m}(t)+\alpha_{f f}^{2} W_{m}(t)=0
$$

So, the entire equation (29) takes the form

$$
\frac{d^{2}}{d t^{2}} W_{m}(t)+\alpha_{f f}^{2} W_{m}(t)=\frac{M g}{\mu m \pi B_{m 5}}\left[-\cos m \pi+\cos \frac{m \pi c t}{L}\right]
$$

Employing Laplace transformation technique and the convolution theory, one obtain an expression for $\phi_{f}$ and when inverted gives 


$$
V(x, t)=\sum_{m=1}^{\infty} \frac{2 B_{m 5}}{m \pi}\left[\frac{\cos \theta_{m} t \cos \alpha_{f f} t}{\alpha_{f f}^{2}-\theta_{m}^{2}}+\frac{\left(1-\cos \alpha_{f f} t\right)}{\alpha_{f f}}\right] \times \sin \frac{m \pi x}{L}
$$

which represents the traverse displacement response to moving distributed mass moving at constant speed on constant elastic foundation.

\section{Discussion Of The Analytical Solution}

When an undamped system such as this is studied, it is required that phenomenon of resonance is examined. Equation (27) clearly reveals that the simply supported the beam under moving distributed force experience effect when

$$
\alpha_{f}=\frac{m \pi c}{L}
$$

while equation (46) shows the same beam under the action of a moving distributed mass

$$
\alpha_{f f}=\frac{m \pi c}{L}
$$

where

$$
\alpha_{f f}=\alpha_{f}\left[1-\frac{\varepsilon_{m}}{2 \alpha_{f}^{2}}\left(\alpha_{f} H_{a} / 4-\alpha_{f}^{2} H_{g} / 4\right)\right]
$$

Thus,

$$
\alpha_{f}=\frac{\frac{m \pi c}{L}}{\left[1-\frac{\varepsilon_{m}}{2 \alpha_{f}^{2}}\left(\alpha_{f} H_{a} / 4-\alpha_{f}^{2} H_{g} / 4\right)\right]}
$$

Clearly, it is seen that for the same natural frequency, the critical speed for the moving force problem is greater than that of the moving distributed force problem. Thus, the resonance is reached earlier in the moving distributed mass system than in the moving distributed force system.

\subsection{Numerical calculation and discussion of results}

For practical purposes, the foregoing analysis is illustrated by considering a uniform simply supported thin beam resting on constant elastic foundation of length $12.192 \mathrm{~m}$ constant velocity of $3.123 \mathrm{~m} / \mathrm{s}$, $E=2.10924 E+09$ and $I=0.0028769 \#$ respectively. The results are displayed on the various graphs below.

Figure 1 and 2 show the effect of foundation modulli on the dynamic deflection of the uniform thin beam with variable prestress of simply supported boundary condition in both cases of moving distributed force and moving distributed mass respectively. The graphs show that an increase in the foundation modulus decreases the dynamic response of the thin beam.

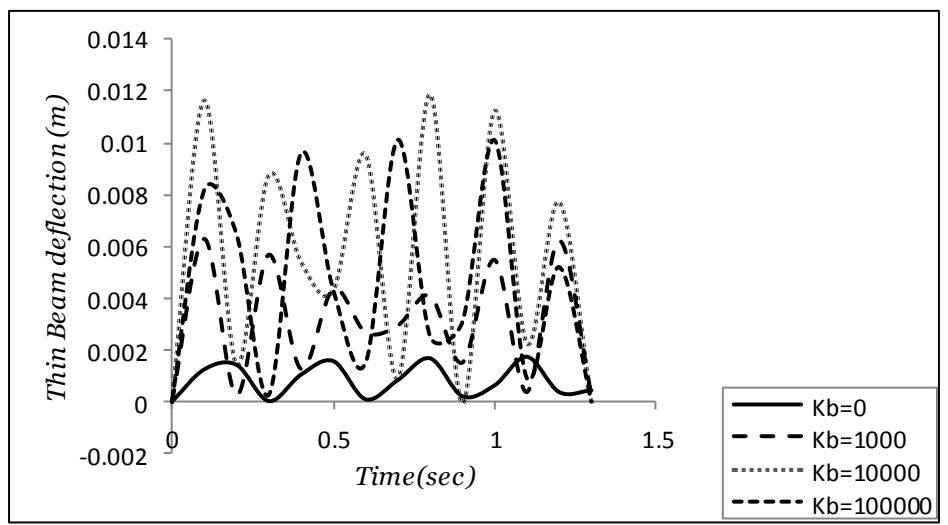

Fig. 1: Deflection profile of thin beam with variable prestress traversed by moving distributed force for various values of foundation modulus. 


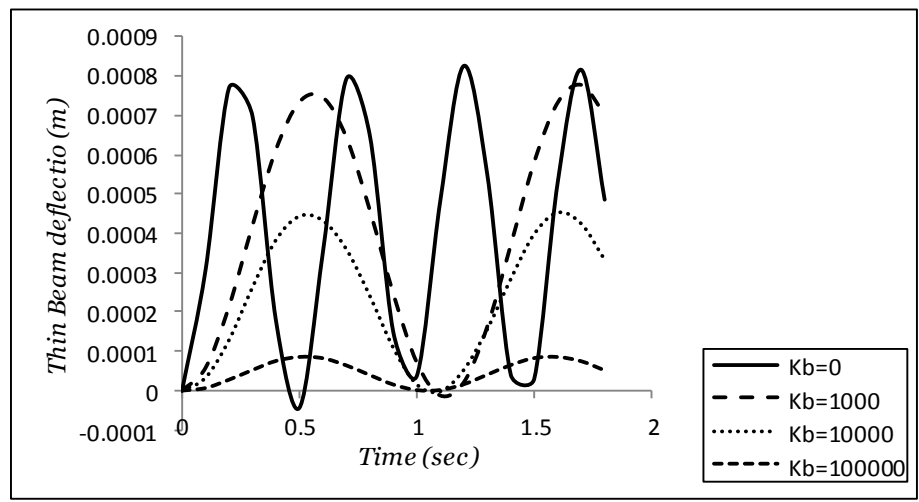

Fig. 2: Deflection profile of thin beam with variable prestress traversed by moving distributed mass for various values of foundation modulus.

Figure 3 and 4 displays the effect of prestress on the dynamic deflection of the uniform thin beam with variable prestress of simply supported boundary condition in both cases of moving distributed force and moving distributed mass respectively. The graphs show that an increase in the prestress decreases the dynamic response of the thin beam.

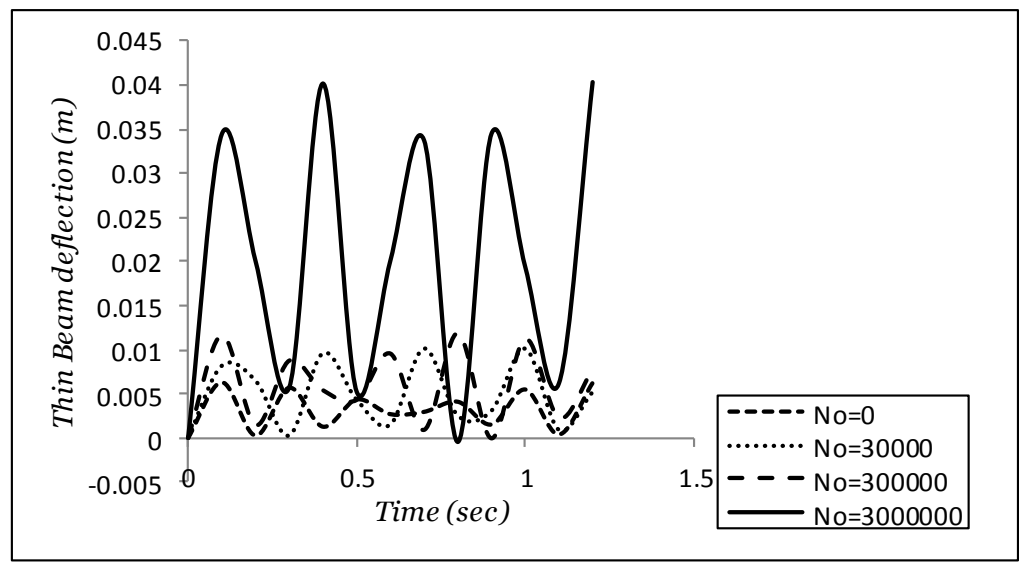

Fig. 3: Desplacement response of thin beam with variable prestress traversed by moving distributed force for various values of prestress $(\mathrm{N})$.

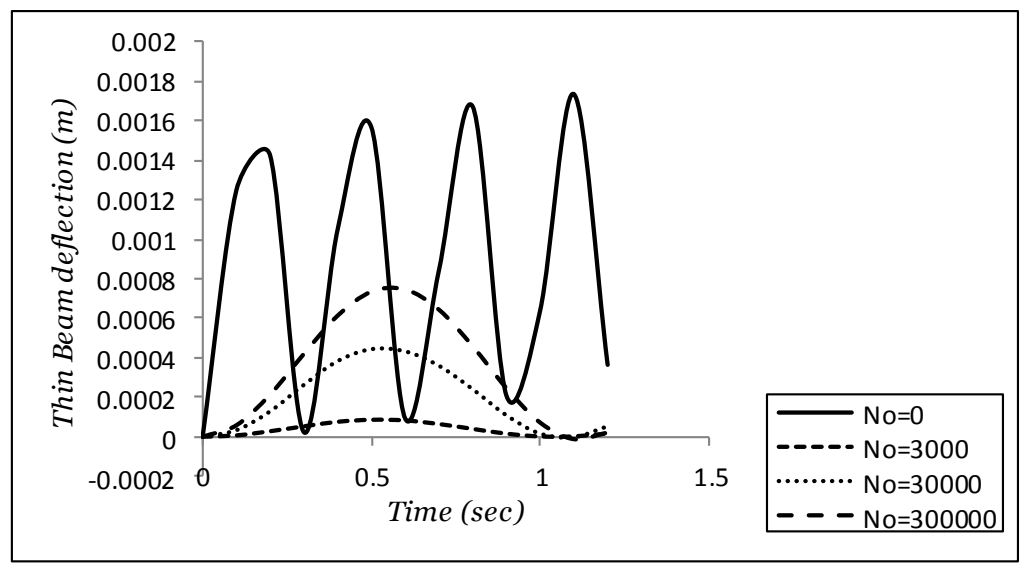

Fig. 4: Desplacement response of thin beam with variable prestress traversed by moving distributed mass for various values of prestress $(\mathrm{N})$.

Figure 5 displays the displacement response of the moving distributed force and moving distributed mass for the thin beam simply supported boundary condition. It is observed that the dynamic amplitude of a moving distributed mass is greater than moving distributed force problem. 


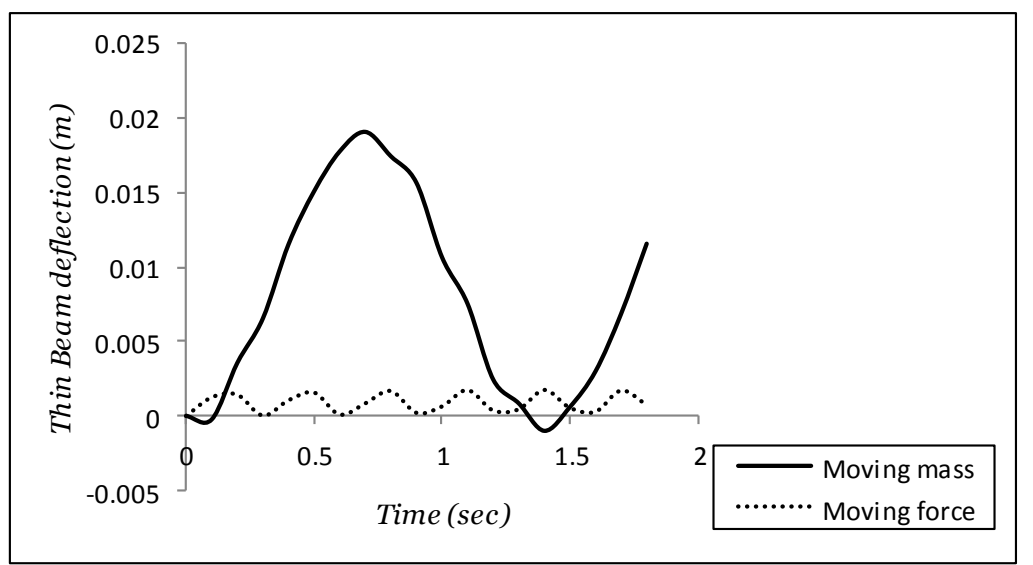

Fig. 5: Comparison of moving distributed force and moving distributed mass of for thin beam with variable prestress traversed by moving distributed load.

\section{Conclusion}

The dynamical analysis of thin beam under a moving distributed load and resting on constant elastic foundation with variable prestress has been presented. The versatile Galerkin's technique was used to reduce the governing fourth order partial differential equations and a modification of Struble's method is used to solve resulting Galerkin's equations. From the numerical analysis, it is noted that as the prestress $N_{b}$ increases, the displacement response of the simply supported beam decrease and for fixed value of prestress $N_{b}$, the response amplitude of simply supported beam decrease as the foundation modulli $K_{b}$ increases. Also, as the mass ratio $\varepsilon_{m}$ increases, the dynamic response of the simply supported thin beam resting on constant elastic foundation under moving distributed load decrease.

Furthermore, for fixed value of foundation modulli $K_{b}$, prestress $N_{b}$ and mass ratio $\varepsilon_{m}$, the displacement response for the moving mass problem is greater than that of moving force problem for the simply supported beam on constant foundation considered.

Finally, for the same natural frequency, the critical speed for the moving force problem is greater than that of the moving mass problem. Therefore, it is deduced that moving force solution is not a save approximation to the moving mass solution.

\section{References}

[1]. H.D. Nelsonand R.A. Conover, Dynamic Stability of a Beam carrying Moving Masses, ASME Journal of Applied Mechanics, ( 93),1971,1003-1006

[2]. [R. Hamada R, Dynamic Analysis of a beam under a moving force. A double Laplace transform solution, Journal of sound and sound and Vibration, (74), 1981, 221- 233.

[3]. S.T. Oni and T.O. Awodola, Dynamic response to moving concentrated masses of uniform Rayleigh beams resting on variable Winkler elastic foundation, Journal of the Nigerian Association of Mathematical Physics,(9), 2005, 151-162.

[4]. K. Achawakorn and T. Jearsiripongkul, Vibration analysis of Exponential Cross-Section Beam Using Galerkin's Method, International Journal of Applied Science and Technology, 2(6), 2012

[5]. J. Jan Tuma, Theory and problems of structural analysis(Schaum's outline series :McGraw-Hill Inc book company, New York, 1967).

[6]. S. T. Oni and S. N. Ogunyebi, Dynamical Analysis of finite prestressed Bernoulli-Euler beams with general boundary conditions under travelling distributed loads, Journal of the Nigerian Association of Mathematical Physics,(12), 2008, 87-102.

[7]. A. N Lowan, On transverse oscillations of beams under the action of moving variable loads, Phil. Mag Ser, 7(19), 1935, 127, 708715 .

[8]. S. Timoshenko, On the correction for shear of the differential equation for transverse vibration of prismatic bar, Phil. Mag. S. 6(41), 1921, 744-776

[9]. S. Timoshenko, D.H Young and W. Weaver Vibration problem inEngineering(Wiley, 448-471, 1974).

[10]. S. T. Oni and T. O. Awodola, Dynamic response under a moving load of an elastically supported non-prismatic Bernoulli-Euler beam on variable elastic foundation, Latin American Journal of Solids and Structures, (7), 2010, 3-20. 